University of Wollongong

Research Online

Australian Institute for Innovative Materials -

Papers

Australian Institute for Innovative Materials

$1-1-2014$

Non-wettable, oxidation-stable, brightly luminescent, perfluorodecyl-capped silicon nanocrystal film

Chenxi Qian

University of Toronto

Wei Sun

University of Toronto

Liwei Wang

Nankai University, University of Toronto

Chanlong Chen

University of Toronto

Kristine Liao

University of Toronto

See next page for additional authors

Follow this and additional works at: https://ro.uow.edu.au/aiimpapers

Part of the Engineering Commons, and the Physical Sciences and Mathematics Commons

Research Online is the open access institutional repository for the University of Wollongong. For further information contact the UOW Library: research-pubs@uow.edu.au 


\title{
Non-wettable, oxidation-stable, brightly luminescent, perfluorodecyl-capped silicon nanocrystal film
}

\begin{abstract}
Here we describe for the first time the synthesis of colloidally stable, brightly luminescent perfluorodecylcapped silicon nanocrystals and compare the properties of solutions and films made from them with those of their perhydrodecyl-capped relatives. The perfluorodecyl capping group compared to the perhydrodecyl capping group yields superior hydrophobicity and much greater resistance to air oxidation, the enhanced electron-withdrawing character induces blue shifts in the wavelength of photoluminescence, and the lower-frequency carbon-fluorine stretching modes disfavor non-radiative relaxation pathways and boost the absolute photoluminescence quantum yield. Together these attributes bode well for advanced materials and biomedical applications founded upon perfluorodecyl-protected silicon nanocrystals.
\end{abstract}

\section{Keywords}

capped, perfluorodecyl, luminescent, brightly, stable, oxidation, wettable, nanocrystal, non, silicon, film

\section{Disciplines}

Engineering | Physical Sciences and Mathematics

\section{Publication Details}

Qian, C., Sun, W., Wang, L., Chen, C., Liao, K., Wang, W., Jia, J., Hatton, B., Casillas, G., Kurylowicz, M., Yip, C. M., Mastronardi, M. L. \& Ozin, G. A. (2014). Non-wettable, oxidation-stable, brightly luminescent, perfluorodecyl-capped silicon nanocrystal film. Journal of the American Chemical Society, 136 (45), 15849-15852.

\section{Authors}

Chenxi Qian, Wei Sun, Liwei Wang, Chanlong Chen, Kristine Liao, Wendong Wang, Jia Jia, Benjamin D. Hatton, Gilberto Casillas, Marty Kurylowicz, Christopher M. Yip, Melanie L. Mastronardi, and Geoffrey A. Ozin 


\title{
Non-Wettable, Oxidation Stable, Brightly-Luminescent, Perfluorodecyl-Capped Silicon Nanocrystal Film
}

\author{
Chenxi Qian, ${ }^{1}$ Wei Sun, ${ }^{1}$ Liwei Wang, ${ }^{1,2}$ Changlong Chen, ${ }^{1,3}$ Kristine Liao, ${ }^{1}$ Wendong Wang, ${ }^{4}$ Jia \\ Jia, ${ }^{1,5}$ Benjamin D. Hatton, ${ }^{5}$ Gilberto Casillas, ${ }^{6}$ Marty Kurylowicz, ${ }^{7}$ Christopher M. Yip, ${ }^{7}$ Melanie L. \\ Mastronardi, ${ }^{1}$ and Geoffrey A. Ozin ${ }^{*}, 1$ \\ ${ }^{1}$ Department of Chemistry, University of Toronto, 8o St. George Street, Toronto, Ontario, $\mathrm{M}_{5} \mathrm{~S} 3 \mathrm{H} 6$, Canada. \\ ${ }^{2}$ Tianjin Key Lab of Metal and Molecule-based Material Chemistry, Department of Chemistry, Nankai University, 94 \\ \# Weijin Road, Tianjin 300071, P R China. \\ ${ }^{3}$ School of Chemistry and Chemical Engineering, University of Jinan, Jinan, Shandong 250022, P R China \\ ${ }^{4}$ Wyss Institute for Biologically Inspired Engineering, Harvard University, Cambridge, MA 02138, USA; School of \\ Engineering and Applied Sciences, Harvard University, Cambridge, MA 02138, USA. \\ ${ }^{5}$ Department of Materials Science \& Engineering, University of Toronto, Wallberg Building, 184 College Street, Suite \\ 140, Toronto, Ontario, $\mathrm{M}_{5} \mathrm{~S}_{3} \mathrm{E}_{4}$, Canada \\ ${ }^{6}$ Electron Microscopy Centre, University of Wollongong, New South Wales 2500, Australia. \\ ${ }^{7}$ Department of Biochemistry, University of Toronto, Toronto, Ontario, Canada; Terrence Donnelly Centre for \\ Cellular and Biomolecular Research, University of Toronto, Toronto, Ontario, Canada.
}

Supporting Information Placeholder

\begin{abstract}
Here we describe for the first time the synthesis of colloidally-stable, brightly-luminescent perfluorodecylcapped silicon nanocrystals and compare the properties of solutions and films with the perhydrodecyl-capped relative. The perfluorodecyl capping group compared to the perhydrodecyl capping group yields superior hydrophobicity and much greater resistance to air oxidation, the enhanced electron withdrawing character induces blue shifts in the wavelength of photoluminescence and the lower frequency carbon-fluorine stretching modes disfavor non-radiative relaxation pathways and boost the absolute photoluminescence quantum yield. Together these attributes bode well for advanced materials and biomedical applications founded upon perfluorodecyl-protected silicon nanocrystals.
\end{abstract}

The burgeoning research activity on new kinds of nanostructured silicon, made from one of the most abundant and green materials on earth, is striking. ${ }^{1,2}$ This activity transcends the utilization of top-down nanofabricated silicon for integration in the incredible shrinking world of more powerful and faster microelectronics. What is remarkable is the notable intensification of research on bottom-up synthesized forms of nanostructured silicon for everything other than microelectronics. ${ }^{3-6}$ Moreover, the purported nontoxicity $^{7}$ and biocompatibility ${ }^{8,9}$ of nanometer scale silicon allows it to be marketed as a "Green" material in the emerging field of medical theranostics. One can easily find a long list highlighting prominent reports for nanometer scale silicon in the recent literature. The typical synthesis involves a hydrosilylation reaction, which enhances the solutionprocessability of the material. This is also an important route in the field of silicon surface chemistry, exemplified by the pioneering work of Buriak, Veinot, Kortshagen, Swihart, etc. $^{10-14}$

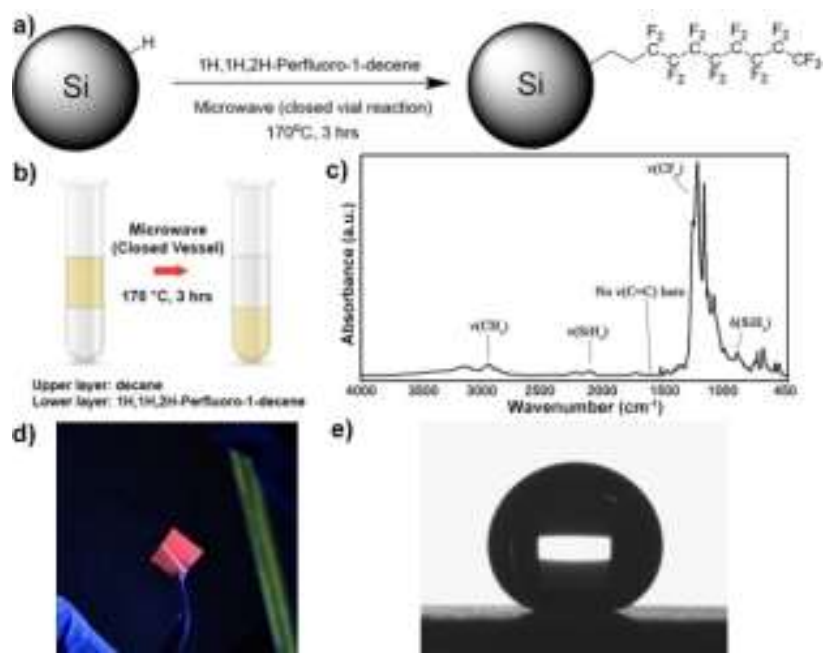

Figure 1. (a,b) Schematic illustration of the synthesis of perfluorodecyl-capped silicon nanocrystals (ncSi:PFD); (c) FT-IR spectrum of ncSi:PFD; (d) Photoluminescence shown by the spin coated ncSi:PFD film on a silicon wafer; (e) Image of a water droplet sitting on the superhydrophobic film composed of ncSi:PFD. 
However, the instability against oxidation has been a major drawback for nanostructured silicon in various applications. There are situations where silicon nanostructured materials could benefit from water resistance and this can in principle be achieved by protecting the surface with super-hydrophobic molecules or polymers, the best documented being based on perfluorohydrocarbons. Specifically, we aim to avoid or ameliorate the situations when silicon nanocrystals are exposed in air and the ambient water accelerates the oxidation of the silicon core, thereby decreasing the photoluminescence quantum yield ${ }^{15,16}$ significantly. The phenomenon of non-wettability stems from the extremely low polarizability of fluorine and the very strong carbon-fluorine single bond which causes very weak van der Waals interactions with perfluorohydrocarbons. While fluoro-substituted alkenes have been chemically anchored to the surface of silicon wafers and porous silicon, ${ }^{17}$ freestanding perfluorodecyl-capped silicon nanocrystals have never been reported before.

In this context, it is interesting to contemplate the effect of covalently grafting perfluoroalkane groups to the surface of silicon nanocrystals rather than perhydroalkanes exclusively reported $^{17-21}$ to date. How would this affect their colloidal stability and wettability, their electronic and optical properties, and the stability against oxidation? In this report we describe for the first time the synthesis and characterization of freestanding perfluorodecyl-capped silicon nanocrystals and measure, compare and discuss the aforementioned behaviors in relation to those of the perhydroalkane analogue. From the point of view of designing and synthesizing solid state materials, it is a demonstration of controlling surface-property relations how surface correlates to and affects the stability of the nanomaterial under certain conditions.

Our synthesis was performed using a microwave reactor, in a closed vial. It is important to note that this reaction system uniquely guarantees a typical hydrosilylation temperature of $170{ }^{\circ} \mathrm{C}$, which is higher than the boiling point of the solvent, $1 \mathrm{H}, 1 \mathrm{H}, 2 \mathrm{H}$-perfluoro-1-decene. The upper layer was decane and the lower was $1 \mathrm{H}, 1 \mathrm{H}, 2 \mathrm{H}$-perfluoro-1decene since they were not miscible. The reaction proceeded for 3 hours to achieve sufficient surface coverage. ${ }^{22}$ In order to get an even higher surface ligand density and better colloidal stability, we extended the reaction time to 24 hours. Before the reaction, the hydride terminated silicon nanocrystals were dispersed in the upper layer of decane; after the reaction, they decended to the lower layer of ${ }_{1} \mathrm{H}, 1 \mathrm{H}, 2 \mathrm{H}$-perfluoro-1-decene (Figures 1 and $\mathrm{S} 1$ ). It was observed that at $170{ }^{\circ} \mathrm{C}$ the two solvents were miscible. So the hydrosilylation process was actually a homogeneous reaction. The dispersion of the product by an overnight reaction was still colloidally stable after one month. After the hydrosilylation, the surface of the silicon nanocrystal was covalently bonded by tetrahydro-perfluorocarbon chains, which was confirmed by Fourier transform infrared (FTIR) spectroscopy (Figure 1c) and X-ray photoelectron spectroscopy (XPS) (Figure S3). The perfluorodecyl capped silicon nanocrystals (ncSi:PFD) were $3-5 \mathrm{~nm}$ in diameter, which is supported by the HR-STEM images (Figure $\mathrm{S}_{4}$ ) and AFM data (Figure S8).

We spin coated the ncSi:PFD onto a single crystal silicon wafer surface. The wafer surface was pretreated ${ }^{23}$ with $1 \mathrm{H}, 1 \mathrm{H}$,
2H, 2H-perfluorooctylsilane (PFOTS) to facilitate the anchoring of the perfluorocarbon capped silicon nanocrystals (Figure S2). Through this method, we obtained uniform films with almost full surface coverage by the ncSi:PFD. A hierarchical structure of the film can be seen through the SEM and AFM images (Figures $\mathrm{S}_{5}$, S8). The ncSi:PFD film showed bright photoluminescence (Figures id and $2 \mathrm{~b}$ ). The surface of the film as prepared was already superhydrophobic after the facile step of spin coating, without any further treatment (Figure 1e). Water droplets roll off the surface easily (Supporting Information (SI), Movies). This surface shows a water contact angle (CA) of $168.3 \pm 0.5^{\circ}$. The advancing and receding CAs of the surface are $167.7^{\circ}$ and $168.8^{\circ}$, respectively (Figure $\mathrm{S}_{7}$ ). Atomic force microscopy (AFM) showed that the film had different RMS roughness at different length scales (Figure S8). The interplay of the multiscale hierarchical structure and low surface free energy contributes to the superhydrophobicity. The multi-scale roughness and the cavities suggest the existence of a CassieBaxter $^{24,25}$ surface state. A very low sliding angle is also an evidence of such a state. A control experiment showed that a PFOTS treated silicon wafer surface did not exhibit an ultrawater-repellent character, with a water CA measured at around $117^{\circ}$. Another control experiment was done with a film composed of decyl group capped silicon nanocrystals (ncSi:D), which showed no superhydrophobicity, either (Figure S6). This comparison confirms the superhydrophobic nature is from the ncSi:PFD film itself and the constituent nanocrystals.
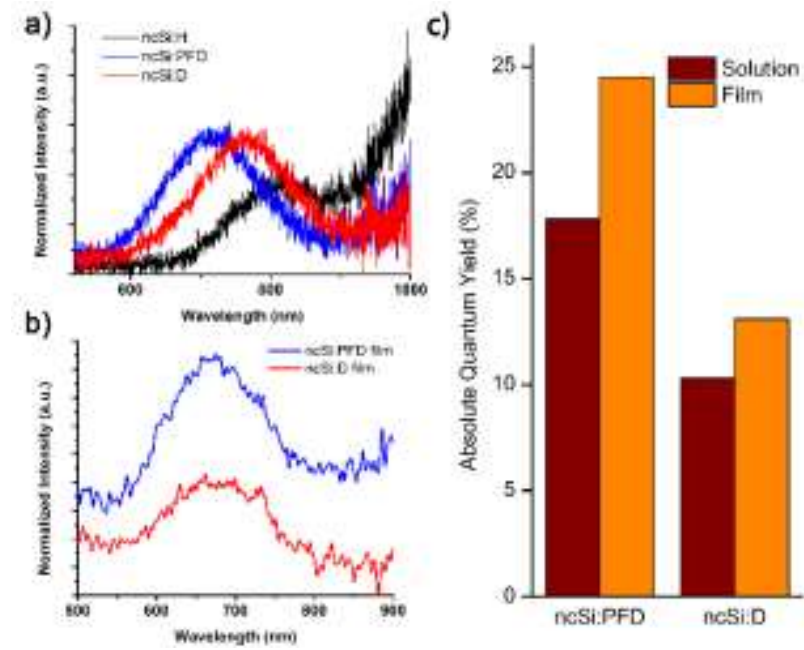

Figure 2. (a) Photoluminescence spectra of hydride, decyl, and perfluorodecyl capped silicon nanocrystals in solution; (b) Photoluminescence spectra of decyl and perfluorodecyl capped silicon nanocrystals in the solid state (film); (c) Comparison between the absolute quantum yield (AQY) of decyl, and perfluorodecyl capped silicon nanocrystals in solution and solid state.

The ncSi:PFD dispersion was brightly photoluminescent and the spectra shifted to the blue compared to the spectra of $\mathrm{ncSi}_{\mathrm{H}} \mathrm{H}$ and $\mathrm{ncSi}: \mathrm{D}$ dispersions (Figure 2a). The emission tails shown at above $900 \mathrm{~nm}$ were from the larger particles within the ensemble. ${ }^{6}$ The blue shift can be explained by the lack of aggregation and less electronic coupling compared to $\mathrm{ncSi}: \mathrm{H}$ dispersions. Due to the lower vibrational levels of ncSi:PFD, they possessed a slightly larger gap and the corresponding spectra shifted further towards the shorter 
wavelength. The PL spectra of ncSi:PFD and ncSi:D films are shown in Figure $2 \mathrm{~b}$. Free from non-radiative solvent relaxations and relaxations via intramolecular rotation, both the ncSi:PFD and ncSi:D films had a higher absolute quantum yield (AQY) than the nanocrystal dispersions (Figure 2c).

A few factors contribute to the higher AQY of ncSi:PFD (dispersion or film) comparing with $\mathrm{ncSi}$ :D (dispersion or film). Firstly, the lower frequency carbon-fluorine stretching mode disfavor non-radiative relaxation pathways and boosts the absolute photoluminescence quantum yield. ${ }^{26,27}$ Specifically, there is less coupling between lower vibrational levels and the electronic excited states, thus less internal conversions occur and non-radiative relaxations are rarer. Secondly, ncSi:PFD are more stable against oxidation and have less oxidation defects (see below). Previous reports ${ }^{28}$ have shown that the introduction of surface oxidation defects would affect the optical properties of silicon nanocrystals and reduce the photoluminescence quantum yield significantly.
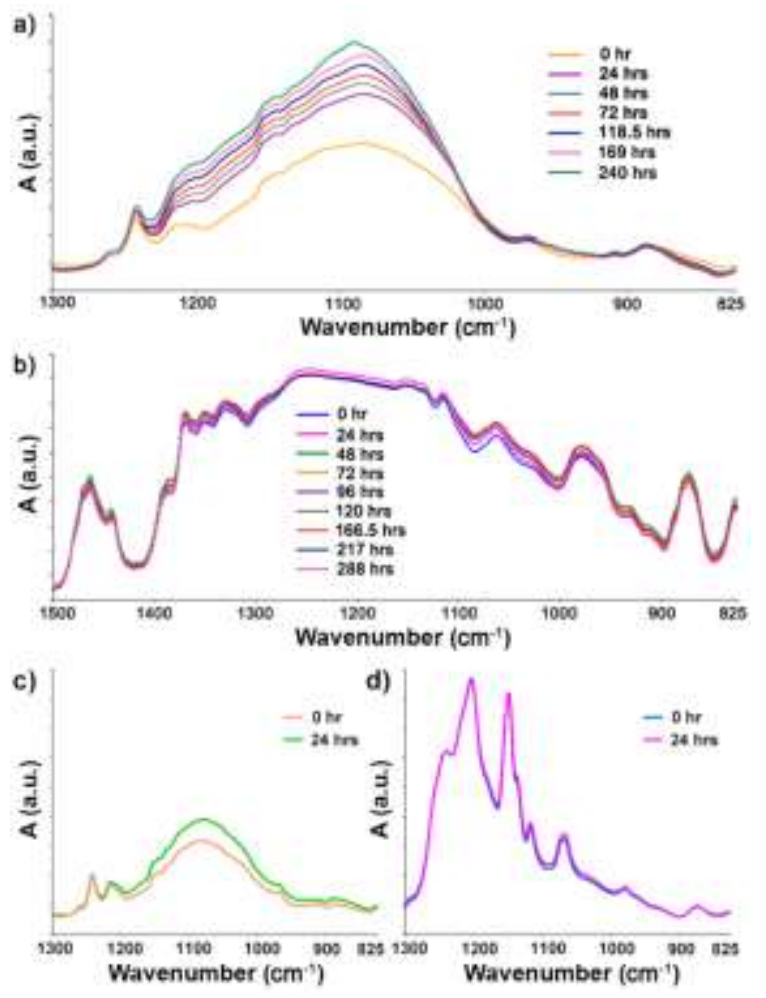

Figure 3. Oxidation trends of silicon nanocrystal films overtime: (a) ncSi:D film in ambient air; (b) ncSi:PFD film in ambient air. (c) ncSi:D film in humid air. (d) ncSi:PFD film in humid air.

Since the as-synthesized perfluorodecyl capped silicon nanocrystals still had remnant terminal hydrides (which was confirmed by FTIR, Figure 1c), they could easily undergo oxidation to hydroxide and oxide in the presence of ambient oxygen and water. It is well-recognized that the oxidation of silicon nanocrystals usually follows the Cabrera-Mott mechanism, ${ }^{29,30}$ as supported by previous investigations. ${ }^{31-33}$ This mechanism describes how a Si crystal surface is oxidized by the simultaneous presence of water and oxygen molecules in the ambient air. Specifically, the polar water molecules approach the surface silanol groups preferentially and aid in the cleavage of $\mathrm{Si}-\mathrm{Si}$ bonds adjacent to the silanol groups.
Then an electron is transferred from the broken bond to an absorbed oxygen molecule. So it can be speculated that the presence of ambient water accelerates the oxidation process, and a water-repellent perfluorodecane surface, on the other hand, can contribute to slowing down such a process.

In order to confirm the expected difference between the ncSi:D and ncSi:PFD films in reactivity, we compared the oxidation trends of the two films (ncSi:D and ncSi:PFD) over a period of time by FTIR. Both films were prepared freshly from the same batch of $\mathrm{ncSi}: \mathrm{H}$, and were exposed to air under the same ambient conditions (25\% R.H.). We investigated the same regions of the FTIR spectra of the ncSi:D and ncSi:PFD, as shown in Figures $3 a$ and $3 b$, respectively. The Si-O-Si mode is centered at $1070 \mathrm{~cm}^{-1}$. The absolute difference in absorption around this region reflects the extent of oxidation. Since the films were prepared freshly at zero hour when little oxidation occurred, a distinct comparison between the peak height in Figures $3 a$ and $3 b$ would be a fair comparison indicating the extent of oxidation. As expected, the result indicates that under the same ambient conditions, the ncSi:D film was oxidized much faster than the ncSi:PFD film was. We also investigated the oxidation of both films exposed to the same humid air ( $94 \%$ R.H.) and compared their reactivity, in which case the ncSi:D film was also oxidized much faster than the ncSi:PFD film, again as expected (Figures $3 \mathrm{c}$ and $3 \mathrm{~d}$ ). It was observed that in a highly humid environment the ncSi:PFD film was still resistant to oxidation.

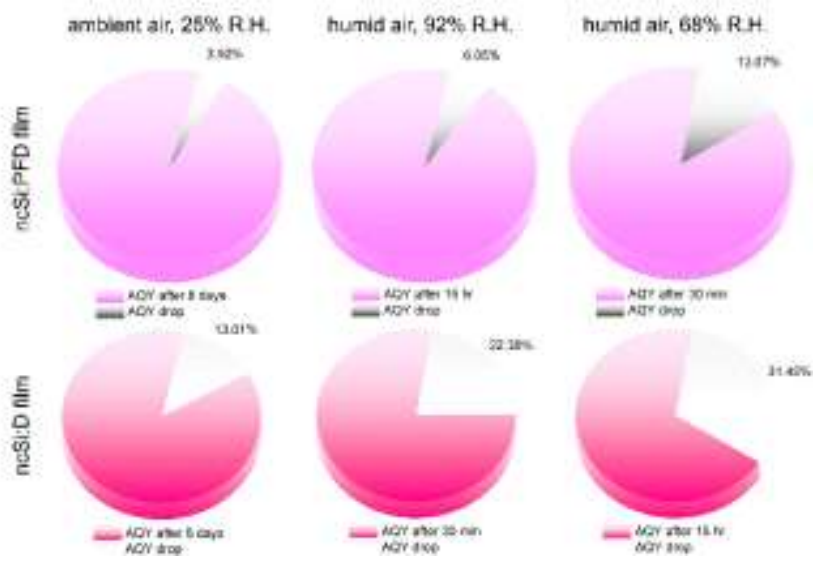

Figure 4. Comparison of the relative drop in absolute quantum yield (AQY) of two films under different conditions (area of the pies reflect the initial absolute values of AQY, $35 \%$ for ncSi:PFD film and $28 \%$ for ncSi:D film): top, ncSi:PFD film; bottom, ncSi:D film; left, exposed to air (R.H. $25 \%$ ) for 8 days; middle, exposed to air (R.H. 92\%) for 30 minutes; right, exposed to air (R.H. 68\%) for 15 hours.

On the other hand, it is important to further investigate the stability of the films by measuring the absolute quantum yield (AQY) after different time of exposure in air with different ambient relative humidity. Figure S9a shows the AQY evolve under different conditions of the film samples made of ncSi:PFD/ncSi:D prepared with a reaction time of 24 hours. The AQY of the ncSi:D film after exposed to air (R.H. $68 \%$ ) for 30 minutes dropped significantly, while that of the ncSi:PFD film dropped relatively less. It was the similar case with exposure to air (R.H. 92\%) for 15 hours. The comparison of the relative drop in AQY is summarized in Figure 4 as pie charts. In ambient air with lower R.H. (25\%), the relative 
AQY drop for both films was much slower, measured at $3.92 \%$ for ncSi:PFD film and $13.01 \%$ for ncSi:D film after 8 days' exposure (absolute value shown in Figure S9b). This comparison (shown in Figure 4) corresponded well with the difference in oxidation rates indicated by the FTIR mentioned above. Clearly, water assisted the oxidation but at the same time passivated the surface as a protection. These two competing effects could explain why in extremely high R.H. the oxidation was slower for both materials compared to those in humid air with moderate R.H. Information about the detailed mechanism is planned for a thorough future study. We also observed a much faster intensity drop of ncSi:D film than ncSi:PFD film after being immersed in distilled water for hours (Figure S10). Clearly, it was undisputable that in any case the ncSi:PFD film was always more stable than ncSi:D film in maintaining its photoluminescence absolute quantum yield.

In summary, freestanding silicon nanocrystals capped by perfluoroalkane chains were synthesized and a higher absolute quantum yield was obtained both for dispersions and films compared to its perhydroalkane analogues. Films of these perfluoroalkane-capped silicon nanocrystals were proven to be superhydrophobic and found to be much more resistant to air oxidation than the perhydroalkane analogue. It is a new, facile and green way of fabricating bi-functional photoluminescent-superhydrophobic nanocrystal surfaces. Future work may include the synthesis of PEG terminated solid lipid particles composed of these ncSi:PFD for a better biocompatibility and biostability in biomedical applications. ${ }^{34}$ Collectively these observations speak well for advanced materials and biomedical uses for these perfluoralkane capped silicon nanocrystals.

\section{ASSOCIATED CONTENT}

\section{Supporting Information}

Chemicals and materials, instruments, experimental details, characterizations including FTIR, HRTEM, XPS, SEM, AFM and superhydrophobicity comparisons between films made by perfluorodecane-capped silicon nanocrystals and the perhydroalkane analogue (including videos). This material is available free of charge via the Internet at http://pubs.acs.org.

\section{AUTHOR INFORMATION}

Corresponding Author

gozin@chem.utoronto.ca

\section{Present Addresses}

Department of Chemistry, University of Toronto, 8o St. George Street, Toronto, Ontario, $\mathrm{M}_{5} \mathrm{~S}_{3} \mathrm{H} 6$, Canada.

Notes

The authors declare no competing financial interests.

\section{ACKNOWLEDGMENT}

GAO is Government of Canada Tier 1 Canada Research Chair in Materials Chemistry. The strong and sustained financial support of this research by the Natural Science and Engineering Research Council of Canada (NSERC) is deeply appreciated. A Premier Research Award in support of this research from the Ministry of Research Innovation (MRI)
Ontario is also greatly acknowledged. Chenxi Qian and Wei Sun are Connaught Scholars and Melanie Mastronardi is a Vanier Graduate Scholar.

\section{REFERENCES}

(1) Priolo, F.; Gregorkiewicz, T.; Galli, M.; Krauss, T. F. Nat. Nanotechnol. 2014, 9, 19.

(2) Mastronardi, M. L.; Henderson, E. J.; Puzzo, D. P.; Ozin, G. A. Adv. Mater. 2012, 24, 5890.

(3) Cheng, K.-Y.; Anthony, R.; Kortshagen, U. R.; Holmes, R. J. Nano Lett. 2010, 10, 1154.

(4) Cheng, K.-Y.; Anthony, R.; Kortshagen, U. R.; Holmes, R. J. Nano Lett. 2011, 11, 1952.

(5) Puzzo, D. P.; Henderson, E. J.; Helander, M. G.; Wang, Z. B.; Ozin, G. A.; Lu, Z. H. Nano Lett. 2011, 11, 1585.

(6) Mastronardi, M. L.; Henderson, E. J.; Puzzo, D. P.; Chang, Y. L.; Wang, Z. B.; Helander, M. G.; Jeong, J. H.; Kherani, N. P.; Lu, Z. H.; Ozin, G. A. Small 2012, 8, 3647 .

(7) Alsharif, N. H.; Berger, C. E. M.; Varanasi, S. S.; Chao, Y.; Horrocks, B. R.; Datta, H. K. Small 2009, 5, 221.

(8) Yang, Z.; Dasog, M.; Dobbie, A. R.; Lockwood, R.; Zhi, Y.; Meldrum, A.; Veinot, J. G. C. Adv. Funct. Mater. 2014, 24, 1345.

(9) Liu, J.; Erogbogbo, F.; Yong, K.-T.; Ye, L.; Liu, J.; Hu, R.; Chen, H.; Hu, Y.; Yang, Y.; Yang, J.; Roy, I.; Karker, N. A.; Swihart, M. T.; Prasad, P. N. ACS Nano 2013, 7, 7303.

(10) Buriak, J. M.; Stewart, M. P.; Geders, T. W.; Allen, M. J.; Choi, H. C.; Smith, J.; Raftery, D.; Canham, L. T. J. Am. Chem. Soc. 1999, 121, 11491.

(11) Huck, L. A.; Buriak, J. M. J. Am. Chem. Soc. 2012, 134, 489.

(12) Kelly, J. A.; Veinot, J. G. C. ACS Nano 2010, 4, 4645.

(13) Mangolini, L.; Jurbergs, D.; Rogojina, E.; Kortshagen, U. J. Lumin. 2006, 121, 327.

(14) Hua, F. J.; Swihart, M. T.; Ruckenstein, E. Langmuir 2005, 21, 6054 .

(15) Littau, K. A.; Szajowski, P. J.; Muller, A. J.; Kortan, A. R.; Brus, L. E. J. Phys. Chem. 1993, 97, 1224.

(16) Wilcoxon, J. P.; Samara, G. A.; Provencio, P. N. Phys. Rev. B 1999, 6o, 2704.

(17) Stewart, M. P.; Buriak, J. M. J. Am. Chem. Soc. 20o1, 123, 7821. (18) Mastronardi, M. L.; Hennrich, F.; Henderson, E. J.; Maier-Flaig, F.; Blum, C.; Reichenbach, J.; Lemmer, U.; Kubel, C.; Wang, D.; Kappes, M. M.; Ozin, G. A. J. Am. Chem. Soc. 2011, 133, 11928.

(19) Kelly, J. A.; Shukaliak, A. M.; Fleischauer, M. D.; Veinot, J. G. C. J. Am. Chem. Soc. 2011, 133, 9564.

(20) Yang, Z.; Iqbal, M.; Dobbie, A. R.; Veinot, J. G. C. J. Am. Chem. Soc. 2013, 135, 17595 .

(21) Buriak, J. M. Chem. Mater. 2014, 26, 763.

(22) Sun, W.; Qian, C.; Mastronardi, M. L.; Wei, M.; Ozin, G. A. Chem. Commun. 2013, 49, 11361.

(23) Sun, W.; Jia, F.; Sun, Z.; Zhang, J.; Li, Y.; Zhang, X.; Yang, B. Langmuir 2011, 27, 8018.

(24) Cassie, A. B. D.; Baxter, S. T Faraday Soc 1944, 40, 0546.

(25) Wang, S.; Jiang, L. Adv. Mater. 2007, 19, 3423.

(26) Lin, S. H. J. Chem. Phys. 1966, 44, 3759.

(27) Becker, R. S.; Pelliccioli, A. P.; Romani, A.; Favaro, G. J. Am. Chem. Soc. 1999, 121, 2104.

(28) Pi, X. D.; Mangolini, L.; Campbell, S. A.; Kortshagen, U. Phys. Rev. $B$ 2007, 75 .

(29) Mott, N. Proc. R. Soc. A 1981, 376, 207.

(30) Cabrera, N.; Mott, N. F. Rep. Prog. Phys. 1948, 12, 163.

(31) Pi, X. D.; Gresback, R.; Liptak, R. W.; Campbell, S. A.; Kortshagen, U. Appl. Phys. Lett. 2oo8, 92.

(32) Liptak, R. W.; Kortshagen, U.; Campbell, S. A. J. Appl. Phys. 2009, 106.

(33) Pereira, R. N.; Rowe, D. J.; Anthony, R. J.; Kortshagen, U. Phys. Rev. B 2011, 83 .

(34) Henderson, E. J.; Shuhendler, A. J.; Prasad, P.; Baumann, V.; Maier-Flaig, F.; Faulkner, D. O.; Lemmer, U.; Wu, X. Y.; Ozin, G. A. Small 2011, 7, 2507. 
Table of Contents Graphic

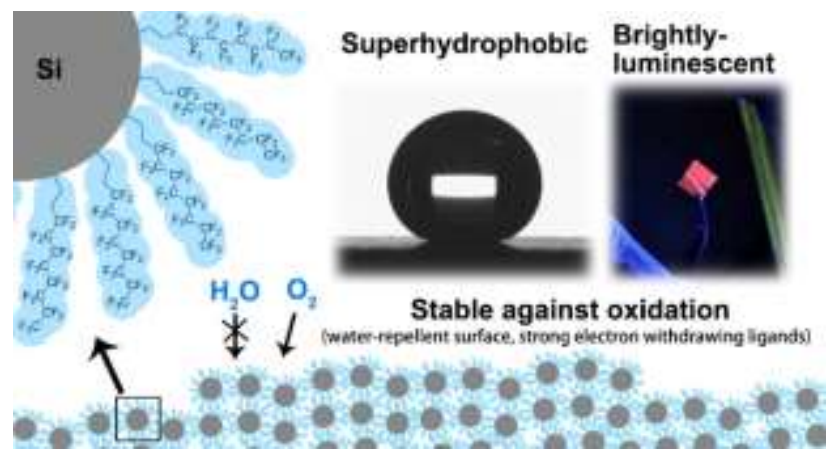

